\title{
CYTOTOXIC AGEN'TS IN THE MANAGEMENT OF IDIOPATHIC THROMBOCYTOPENIC PURPURA
}

\author{
D. Ogston, M.B., Ph.D. (Abdn.), M.R.C.P.E. \\ Lecturer in Medicine
}

\author{
H. W. Fullerton, M.A., M.D.(Abdn.), F.R.C.P., F.R.C.P.E. \\ Professor of Medicine \\ Department of Medicine, University of Aberdeen.
}

THE CONCEPT of auto-immunity and the recognition of diseases resulting from auto-immune processes thas been followed by itrials of the use of the immuno-suppressive properties of cytotoxic drugs in their management. Benefit from their use has been reported in systemic lupus erythematosus (Demeshek and Schwartz, 1960), in auto-immune haemolytic anaemia (Schwartz and Dameshek, 1962), and in active chronic hepatitis (Mackay, Weiden and Ungar, 1964). Richmond, Woodruff, Cumming and Donald (1963) have described a patient with idiopathic thrombocytopenic purpura who relapsed fiollowing splenectomy and failed to respond to corticosteroid therapy, but who had a good response to a combination of thymic irradiation, actinomycin $\mathrm{C}$ and azathioprine. We report two further cases of idiopathic thrombocytopenic purpura to illustrate the value of cytotoxic drugs when other forms of treatment have failed or are contraindicated.

\section{Case Reports}

\section{Case 1:}

A distillery worker, aged 66 , was admitted to Aberdeen Royal Infirmary in January, 1964, with a six-week history of easy bruising and profuse epistaxes. The previous medical history was unremarkable apart from oocasional dyspepsia; a barium meal in 1960 had shown the presence of a duodenal ulcer. There was no history of drug ingestion or of exposure to chemical agents. He was found to have a haemoglobin level of $9.6 \mathrm{~g}$. \%. He was transfused with 2 pints of blood and bleeding points in his nose cauterised. On examination at this time he had numerous petechiae and ecchymoses, most marked on the feet and ankles. The spleen was not palpable and there was no lymphadenopathy. $\mathrm{He}$ was then referred to us for further investigation and treatment. The blood count showed a haemoglobin of $12.3 \mathrm{~g}$. \%, leuoocyte count 7,750/icu.mm., with a normal differential count, and platelets less than $10,000 / \mathrm{cu} . \mathrm{mm}$. Sternal marrow smears showed normal cellularity and an increase in megakaryocytes. The L.E. latex test and examinations for L.E. cells were negative. The serum protein electrophoretic pattern was normal and the sedimentation rate (Westergren) was 7 $\mathrm{mm} . / 1 \mathrm{st}$ hour. Treatment with prednisone $(60$ mg./day) was started on 11.1.64. This produced a moderate increase in the platelet count to $115,000 /$ cu.mm., but on reducing the dosage the count began to fall again. The decision was therefore made to proceed with splenectomy. Under ACTH and cortisone cover the spleen, weighing $150 \mathrm{~g}$. and showing no specific abnormality on histological examination, was removed on 6.3.64. Splenectomy was followed by the expected steep rise in the platelet count to $424,000 /$ cu.mm.

The patient remained well until December, 1964, when he had a further epistaxis and spontaneous bruises appeared. On examination he had numerous petechiae, especially on the legs, and large ecchymoses on both upper arms. The platelet count was $18,000 /$ cu.mm., and treatment with prednisone $(40$ mg./day) was started. The platelet counts from this point onwards are shown in Fig. 1. On admission to hospital on 5.1.65. ACTH gel (20 units/day) was added. In view of the slow response of the platelet count to this therapy it was decided to try the effect of a cytotoxic agent and azathioprine ('Imuran') in a dosage of $150 \mathrm{mg}$./day was started on 16.1.65. The platelet count continued to rise and the dose of prednisone was reduced. On 30.1 .65 he appeared pale, the haemoglobin level had fallen to $6.7 \mathrm{~g}$. \%, and the stool orthotolidine was found to be strongly positive for oocult blood. On questioning he admitted to some dyspepsia over the previous few days. Both prednisone and azathioprine were stopped and he was itransfused with 4 pints of blood. Two days later azathioprine was restarted and on this drug and $\mathrm{ACTH}$, and later on azathioprine alone, his platelet count rose to a peak of $389,000 / \mathrm{cu} . \mathrm{mm}$., and he was discharged from hospital on a maintenance dose of $50 \mathrm{mg}$. azathioprine daily. Three weeks later the patient was forced to stop the drug because of anorexia and nausea, and these symptoms reappeared on restarting the drug. All treatment was discontinued and over the next few months the platelet count slowly fell. When the platelet count had fallen to less than $100,000 /$ cu.mm., he was asked to restart azathioprine. The first tablet induced severe retching and this was repeated on each occasion when he took even a portion of a tablet. 6-mencaptopurine in a dosage of $150 \mathrm{mg}$./day was well-tolerated and induced a steady rise in the platelet count to $244,000 /$ cu.mm., when the dose was reduced because of a fall in the neutrophil polymorph count. Four months after the cessation of all therapy the platelet count was $138,000 / \mathrm{cu} . \mathrm{mm}$., and he had no further haemorrhagic features.

\section{Case 2:}

In 1944, when aged 23, this housewife developed 


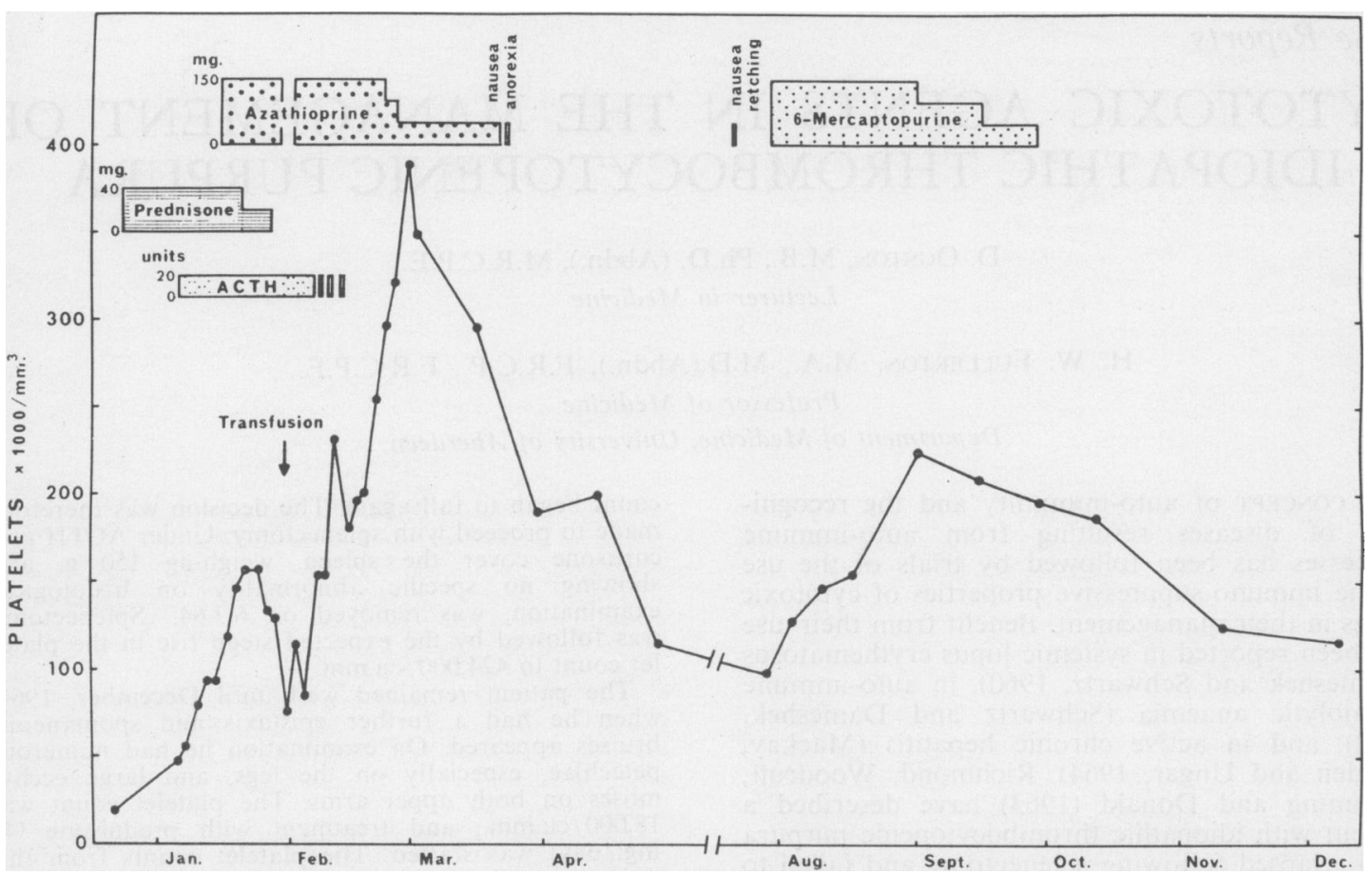

Fig. 1.- Case 1.

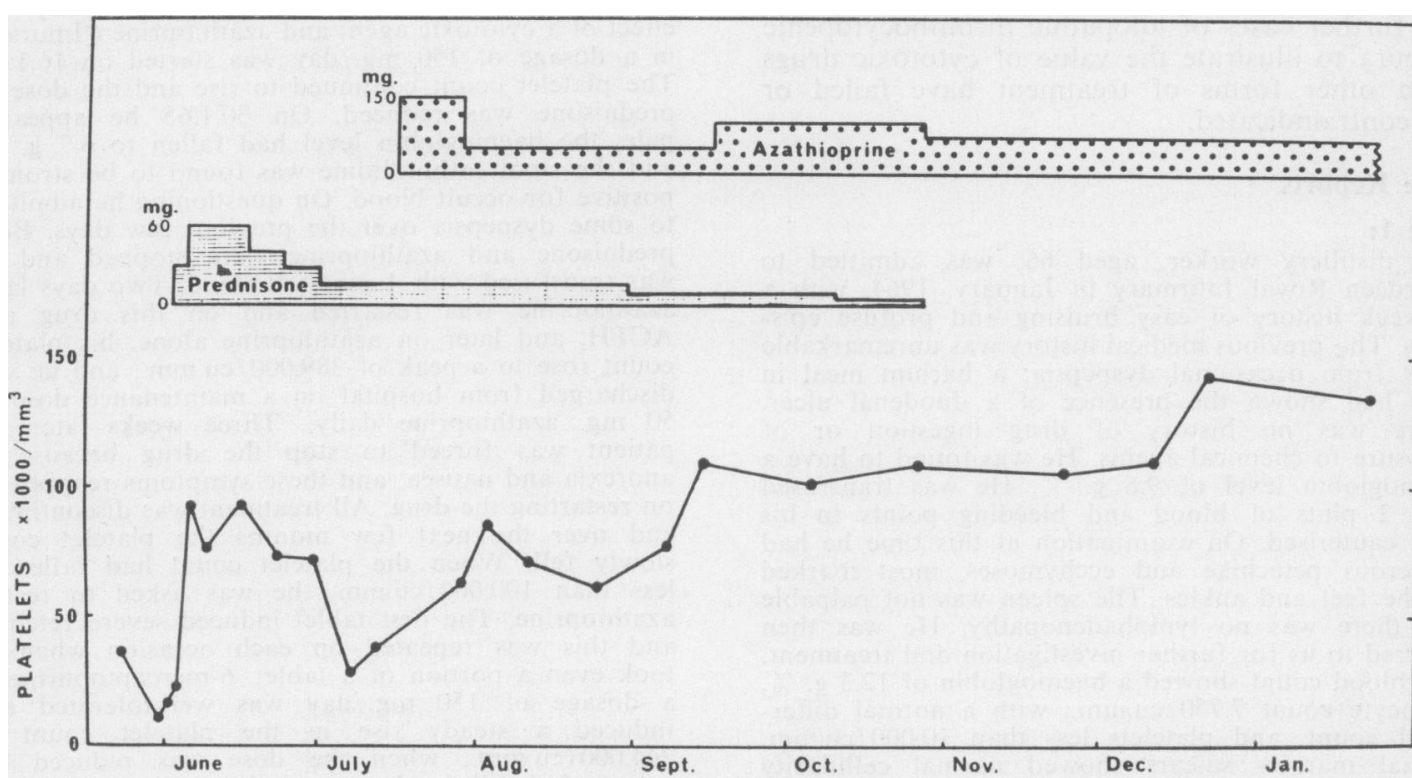

Fig. 2.- Case 2.

typical rheumatoid arthritis affecting the hands, shoulders, knees, ankles, and temporomandibular joints. The arthritis has now been quiescent for a number of years. In 1951 she presented with corneal ulcers secondary to keratitis sicca and was considered to be an example of Sjögrens syndrome. This has requirement intermittent treatment with local corticosteroid and antibiotic since that time. 
In 1962 , at the age of 40 , she was admitted to Aberdeen Royal Infirmary under the care of Dr. I. Gordon, with an 11-week history of tiredness and anorexia following an attack of bronchitis. Apart from mucosal pallor and rheumatoid deformity of the hands there were no significant physical findings. The sedimentation rate (Westergren) was markedly elevated at 87 to $96 \mathrm{~mm}$./1st hour. Serum protein electrophoresis showed an increase in $\gamma$-globulin and a slight increase in $\alpha_{2}$-globulin. The R. A. Latex and Rose-Waaler tests were positive (D.A.T. 1/16). She had a moderate anaemia (Hb. $9.5 \mathrm{~g} . \%$ ), the red cells showing hypochromia, and the white cell count varied between 2,000 and $4,000 / \mathrm{cu} . \mathrm{mm}$., with a relative lymphocytosis. The platelet count was $128,000 /$ cu.mm., and the reticulocyte count was less than $1 \%$. The sternal marrow was hypercellular with some increase in plasma cells. She was transfused with 6 pints of blood and started on oral iron. She was kept under periodic review over the next two years; the erythocyte sedimentation rate remained elevated but the haemoglobin was maintained between 10.8 and $12.3 \mathrm{~g}$. \%. At review in May, 1965, she gave a 3-month history of episodic epistaxes and the platelet count was found to be $37,000 / \mathrm{cu} . \mathrm{mm}$. She was accordingly re-admitted under the care of Dr. I. Gordon. There were no physical findings apart from a few ecchymoses. The spleen was not palpable and the abdominal X-ray confirmed the absence of splenic enlargement. Investigations showed that the serum had anti-nuclear activity while the thyroid antibody tests were negative. Dr. K. L. G. Goldsmith kindly tested the serum and found that the indirect anti-globulin consumption test for complete platelet antibodies was positive; the test for platelet agglutinins was negative, the haemoglobin was $13.4 \mathrm{~g} \%$. and the leucyte count $1,900 / \mathrm{cu} . \mathrm{mm}$. Further platelet counts were 11,000 and $17,000 /$ cu.mm., and she was therefore started on prednisone. This induced a moderate rise in the platelet count, but when the dose was reduced the count fell gain (see Fig. 2). Azathioprine was introduced while the dose of prednisone was $15 \mathrm{mg}$. daily. The platelet count showed a satisfactory increase while prednisone and azathioprine were combined and this has been maintained after the cessation of prednisone. The leucocyte count rose to $11,300 /$ cu.mm., after the start of prednisone and has remained between 3,150 and $6,750 / \mathrm{cu} . \mathrm{mm}$., while on azathioprine. There have been no fresh haemorrhagic features.

\section{Discussion}

Chronic idiopathic thrombocytopenic purpura usually responds to splenectomy, the incidence of lasting remissions varying in reported series from about 50\% (Stefanini and Dameshek, 1962) to over $80 \%$ (Carpenter, Wintrobe, Fuller, Haut and Cartwright, 1959; Doan, Bouroncle and Wiseman, 1960). Further treatment for the remainder who relapse has, until recently been confined to corticosteroids. These drugs may induce a remission with freedom from haemorrhagic features, but they may fail or their continued use may be contraindicatd because of serious side-effects. It is in this latter group of patients that the beneficial action of other immuno-suppressive agents would be particularly welcome and Case 1, in whom activation of a peptic ulcer by corticosteroids with severe haemorrhage forced the discontinuation of this treament, provides an illustration of the value of cytotoxic drugs in this situation. In Case 2, following failure of corticosteroids, azathioprine was used in preference to splenectomy because of the presence of several auto-immune diseases.

The association of a number of the diseases thought to result from an auto-immune process in a single patient is well-recognised. Case 2 provides a further example of these associations with rheumatoid arthritis, Sjögren's syndrome and finally thrombocytopenic purpura.

The relative incidence of side-effects between 6-mercaptopurine and azathioprine is not yet defined. Since azathioprine is a derivative of 6mercaptopurine it is of interest that azathioprine produced gastric symptoms in Case 1 in a manner suggestive of a sensitivity reaction whereas 6mercaptopurine was subsequently well-tolerated. No side-effects have occurred during the six months treatment with azathioprine in Case 2.

\section{Summary}

Two cases of idiopathic thrombocytopenia are described to illustrate the value of cytotoxic drugs used as immuno-suppressive agents in its management.

We wish to thank Dr. I. Gordon for the opportunity to study Case 2.

\section{REFERENCES}

Carpenter, A. F., Wintrobe, M. M., Fuller, E. A., HaUt, A., and CarTwright, G. E. (1959): Treatment of Idiopathic Thrombocytopenic Purpura, J. Amer. med. Ass., 171, 1911.

DamesheK, W., and SchwarTZ, R. (1960): Treatment of Certain "Auto-immune" Diseases with Antimetabolites; a Preliminary Report, Trans. Ass. Amer. Phycns., 73, 113.

Doan, C. A., Bouroncle, B. A., and Wiseman, B. K. (1960): Idiopathic and Secondary Thrombocytopenic Purpura: Clinical Study and Evaluation of 381 Cases Over a Period of 28 Years, Ann. intern. Med., 53, 861.

MACKAY, I. R., WeIDEN, S., and UnGaR, B. (1964): Treatment of Active Chronic Hepatitis and Lupoid Hepatitis with 6-Mercaptopurine and Azathioprine, Lancet, i, 899.

Richmond, J., WoOdRufF, M. A. F., Cumming, R. A., and DonaLD, K. W. (1963): A Case of Idiopathic Thrombocytopenia and Auto-immune Haemolytic Anaemia Treated by Thymic Irradiation and by Administration of Imuran and Actinomycin-C, Lancet, ii, 125.

SCHWARTZ, R., and DAMESHEK, W. (1962): The Treatment of Autoimmune Haemolytic Anaemia with 6-Mercaptopurine and Thioguanine, Blood, 19, 483.

STEPANini, M., and DamesheK, W. (1962): The Haemorrhagic Disorders. A Clinical and Therapoutic Approach. 2nd Ed., p. 312. Now York: Grune and Stratton. 